Journal of Algebra Combinatorics Discrete Structures and Applications

\title{
Decomposition of product graphs into sunlet graphs of order eight*
}

\section{Kaliappan Sowndhariya, Appu Muthusamy}

\begin{abstract}
For any integer $k \geq 3$, we define sunlet graph of order $2 k$, denoted by $L_{2 k}$, as the graph consisting of a cycle of length $k$ together with $k$ pendant vertices, each adjacent to exactly one vertex of the cycle. In this paper, we give necessary and sufficient conditions for the existence of $L_{8}$-decomposition of tensor product and wreath product of complete graphs.
\end{abstract}

2010 MSC: $05 C 51$

Keywords: Graph decomposition, Product graphs, Corona graph, Sunlet graph

\section{Introduction}

All graphs considered here are finite, simple and undirected. For the standard graph-theoretic terminology the readers are referred to [7]. A cycle of length $k$ is called $k$-cycle and it is denoted by $C_{k}$. Let $K_{m}$ denotes the complete graph on $m$ vertices and $K_{m, n}$ denotes the complete bipartite graph with $m$ and $n$ vertices in the parts. We denote the complete $m$-partite graph with $n_{1}, n_{2}, \ldots, n_{m}$ vertices in the parts by $K_{n_{1}, n_{2}, \ldots, n_{m}}$. For any integer $\lambda>0, \lambda G$ denotes the graph consisting of $\lambda$ edge-disjoint copies of $G$. The complement of the graph $G$ is denoted by $\bar{G}$. The subgraph of $G$ induced by $S \subseteq V(G)$ is denoted as $\langle S\rangle$. For any two graphs $G$ and $H$ of orders $m$ and $n$, respectively, the corona product $G \odot H$ is the graph obtained by taking one copy of $G, m$ copies of $H$ and then joining the $i$ th vertex of $G$ to every vertex in the $i$ th copy of $H$. We define the sunlet graph $L_{2 k}$ with $V\left(L_{2 k}\right)=\left\{x_{1}, x_{2}, \ldots, x_{k}, x_{k+1}, x_{k+2}, \ldots, x_{2 k}\right\}$ and $E\left(L_{2 k}\right)$ $=\left\{x_{i} x_{i+1}, x_{i} x_{k+i} \mid i=1,2, \ldots, k\right.$ and subscripts of the first term is taken addition modulo $\left.k\right\}$. We denote it by $L_{2 k}=\left(\begin{array}{cccc}x_{1} & x_{2} & \ldots & x_{k} \\ x_{k+1} & x_{k+2} & \ldots & x_{2 k}\end{array}\right)$. Clearly, $C_{k} \odot K_{1} \cong L_{2 k}$.

For two graphs $G$ and $H$, their tensor product $G \times H$ and lexicographic or wreath product $G \otimes H$ have

* This work was supported by Department of Science and Technology, University Grant Commission, Government of India.

Kaliappan Sowndhariya(Corresponding Author), Appu Muthusamy; Department of Mathematics, Periyar University, Salem, Tamil Nadu, India (email: sowndhariyak@gmail.com,ambdu@yahoo.com). 
the same vertex set $V(G) \times V(H)=\{(g, h): g \in V(G)$ and $h \in V(H)\}$ and their edge sets are defined as follows:

$E(G \times H)=\left\{(g, h)\left(g^{\prime}, h^{\prime}\right): g g^{\prime} \in E(G)\right.$ and $\left.h h^{\prime} \in E(H)\right\}$,

$E(G \otimes H)=\left\{(g, h)\left(g^{\prime}, h^{\prime}\right): g g^{\prime} \in E(G)\right.$ or $g=g^{\prime}$ and $\left.h h^{\prime} \in E(H)\right\}$. It is well known that the above products are associative and distributive over edge-disjoint unions of graphs and the tensor product is commutative. It is easy to observe that $K_{m} \otimes \overline{K_{n}} \cong K_{n, n, \ldots n}(m$ times $)$.

We shall use the following notation throughout the paper. Let $G$ and $H$ be simple graphs with vertex sets $V(G)=\left\{x_{1}, x_{2}, \ldots, x_{n}\right\}$ and $V(H)=\left\{y_{1}, y_{2}, \ldots, y_{m}\right\}$. Then for our convenience, we write $V(G) \times V(H)=\bigcup_{i=1}^{n} X_{i}$, where $X_{i}$ stands for $x_{i} \times V(H)$. Further, in the sequel, we shall denote the vertices of $X_{i}$ as $\left\{x_{i}^{j} \mid 1 \leq j \leq m\right\}$, where $x_{i}^{j}$ stands for the vertex $\left(x_{i}, y_{j}\right) \in V(G) \times V(H)$.

A labeling of a graph $G$ with $n$ edges is an injection $\rho$ from $V(G)$, the vertex set of $G$, into a subset $S \subseteq Z_{2 n+1}$, the additive group $Z_{2 n+1}$. The length of an edge $e=x y$ with end vertices $x$ and $y$ is defined as $l(x y)=\min \{\rho(x)-\rho(y), \rho(y)-\rho(x)\}$. Note that the subtraction is performed in $Z_{2 n+1}$ and hence $1 \leq l(e) \leq n$. If the length of the $n$ edges are distinct and is equal to $\{1,2, \ldots, n\}$, then $\rho$ is a rosy labeling; moreover, if $S \subseteq\{1,2, \ldots, n\}$, then $\rho$ is a graceful labeling. A graceful labeling is said to be an $\alpha$-labeling if there exists a number $\alpha_{0}$ with the property that for every edge $e=x y$ in $G$ with $\alpha(x)<\alpha(y)$ it holds that $\alpha(x) \leq \alpha_{0}<\alpha(y)$.

By a decomposition of a graph $G$, we mean a list of edge-disjoint subgraphs of $G$ whose union is $G$. For a graph $G$, if $E(G)$ can be partitioned into $E_{1}, E_{2}, \ldots, E_{k}$ such that the subgraph induced by $E_{i}$ is $H_{i}$, for all $i, 1 \leq i \leq k$, then we say that $H_{1}, H_{2}, \ldots, H_{k}$ decompose $G$ and we write $G=H_{1} \oplus H_{2} \oplus \ldots \oplus H_{k}$, since $H_{1}, H_{2}, \ldots, H_{k}$ are edge-disjoint subgraphs of $G$. For $1 \leq i \leq k$, if $H_{i} \cong H$, we say that $G$ has a $H$ decomposition.

Study of $H$-decomposition of graphs is not new. Many authors around the world are working in the field of cycle decomposition [4, 8, 9, 21, 22], path decomposition [24, 25], star decompositon [19, 23, 26, 27] and Hamilton cycle decomposition $[2,3,15,16]$ problems in graphs. Here we consider the sunlet decomposition of product graphs. Anitha and Lekshmi $[5,6]$ proved that $n$-sun decomposition of complete graph, complete bipartite graph and the Harary graphs. Liang and Guo [17, 18] gave the existence spectrum of a $k$-sun system of order $v$ as $k=2,4,5,6,8$. Fu et. al. [12, 13] obtained that 3-sun decompositions of $K_{p, p, r}, K_{n}$ and embed a cyclic steiner triple system of order $n$ into a 3 -sun system of order $2 n-1$, for $n=1(\bmod 6)$. Further they obtained $k$-sun system when $k=6,10,14,2^{t}$, for $t>1$. Fu et. al. [11] obtained the existence of a 5-sun system of order $v$. Gionfriddo et.al. [14] obtained the spectrum for uniformly resolvable decompositions of $K_{v}$ into 1-factor and $h$-suns. Akwu and Ajayi [1] obtained the necessary and sufficient conditions for the existence of decomposition of $K_{n} \otimes \overline{K_{m}}$ and $\left(K_{n}-I\right) \otimes \overline{K_{m}}$, where $I$ denote the 1-factor of a complete graph into sunlet graph of order twice the prime.

In this paper, we obtain the decomposition of some product graphs into sunlet graphs of order eight which is the least even order not proved so far for product graphs, which motivate us to consider this problem. In Section 2, we obtain the necessary and sufficient conditions for the existence of $L_{8}$-decomposition of complete bipartite graphs with part size $m$ and $n$. In Section 3, we obtain the necessary and sufficient conditions for the existence of $L_{8}$-decomposition of tensor product of complete graphs. In Section 4 , we obtain the necessary and sufficient conditions for the existence of $L_{8}$-decomposition of complete multipartite graphs with uniform part size.

To prove our results, we state the following:

Theorem 1.1. [20] For all $n \geq 3, C_{n} \odot K_{1}$ is an $\alpha$-labeling.

Theorem 1.2. [10] Let $G$ be a graph with $n$ edges. If $G$ admits a rosy labeling, then it decomposes $K_{2 n+1}$; if $G$ admits an $\alpha$-labeling, then it decomposes $K_{2 n p+1}$ for every $p>0$.

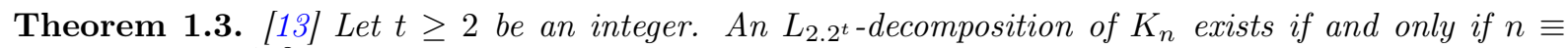
0 (or) $1\left(\bmod 2^{t+2}\right)$.

Remark 1.4. If $n \equiv 0(\bmod 4)$, then $K_{4, n}$ can be decomposed as copies of $K_{4,4}$ and $L_{8}$-decomposition of $K_{4,4}$ is shown in below figure. Therefore $L_{8}$-decomposition exists in $K_{4, n}$ for $n$ is a multiple of 4. 

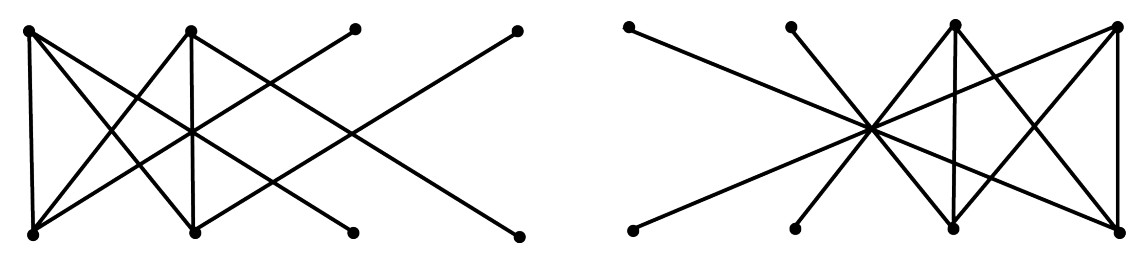

Figure 1. $L_{8}$ - decomposition of $K_{4,4}$.

\section{2. $\quad L_{8}$ - decomposition of $K_{m, n}$}

Now we obtain the necessary and sufficient conditions for the existence of an $L_{8}$-decomposition of $K_{m, n}$ as follows. Let the vertices of $K_{m, n}$ be $\left\{x_{1}, x_{2}, \ldots, x_{m}, y_{1}, y_{2}, \ldots, y_{n}\right\}$.

Lemma 2.1. There exists an $L_{8}$ - decomposition of $K_{8,6}$.

Proof. We exhibit the $L_{8^{-}}$- decomposition of $K_{8,6}$ as follows:

$\left(\begin{array}{llll}x_{1} & y_{1} & x_{2} & y_{2} \\ y_{3} & x_{5} & y_{4} & x_{6}\end{array}\right),\left(\begin{array}{llll}x_{3} & y_{3} & x_{4} & y_{4} \\ y_{5} & x_{2} & y_{6} & x_{1}\end{array}\right),\left(\begin{array}{llll}x_{5} & y_{5} & x_{6} & y_{6} \\ y_{3} & x_{1} & y_{4} & x_{2}\end{array}\right),\left(\begin{array}{llll}x_{7} & y_{5} & x_{8} & y_{6} \\ y_{1} & x_{2} & y_{2} & x_{1}\end{array}\right)$

$\left(\begin{array}{llll}x_{7} & y_{3} & x_{8} & y_{4} \\ y_{2} & x_{6} & y_{1} & x_{5}\end{array}\right),\left(\begin{array}{llll}x_{3} & y_{1} & x_{4} & y_{2} \\ y_{6} & x_{6} & y_{5} & x_{5}\end{array}\right)$.

Lemma 2.2. There exists an $L_{8}$ - decomposition of $K_{8,7}$.

Proof. We exhibit the $L_{8}$ - decomposition of $K_{8,7}$ as follows:

$\left(\begin{array}{llll}x_{1} & y_{1} & x_{2} & y_{2} \\ y_{5} & x_{4} & y_{7} & x_{7}\end{array}\right),\left(\begin{array}{llll}x_{3} & y_{3} & x_{4} & y_{4} \\ y_{2} & x_{1} & y_{5} & x_{2}\end{array}\right),\left(\begin{array}{llll}x_{5} & y_{5} & x_{6} & y_{6} \\ y_{4} & x_{3} & y_{7} & x_{1}\end{array}\right),\left(\begin{array}{llll}x_{7} & y_{1} & x_{8} & y_{7} \\ y_{3} & x_{6} & y_{6} & x_{5}\end{array}\right)$

$\left(\begin{array}{llll}x_{7} & y_{4} & x_{8} & y_{5} \\ y_{6} & x_{1} & y_{3} & x_{2}\end{array}\right),\left(\begin{array}{llll}x_{3} & y_{6} & x_{4} & y_{7} \\ y_{1} & x_{2} & y_{2} & x_{1}\end{array}\right),\left(\begin{array}{llll}x_{5} & y_{2} & x_{6} & y_{3} \\ y_{1} & x_{8} & y_{4} & x_{2}\end{array}\right)$.

Lemma 2.3. There exists an $L_{8}$ - decomposition of $K_{8,9}$.

Proof. We exhibit the $L_{8}$ - decomposition of $K_{8,9}$ as follows:

$\left(\begin{array}{llll}x_{1} & y_{1} & x_{2} & y_{2} \\ y_{3} & x_{6} & y_{4} & x_{7}\end{array}\right),\left(\begin{array}{llll}x_{3} & y_{3} & x_{4} & y_{4} \\ y_{8} & x_{5} & y_{5} & x_{6}\end{array}\right),\left(\begin{array}{llll}x_{5} & y_{5} & x_{6} & y_{6} \\ y_{1} & x_{3} & y_{7} & x_{4}\end{array}\right),\left(\begin{array}{llll}x_{7} & y_{7} & x_{8} & y_{8} \\ y_{5} & x_{4} & y_{6} & x_{5}\end{array}\right)$,

$\left(\begin{array}{llll}x_{3} & y_{1} & x_{4} & y_{9} \\ y_{6} & x_{8} & y_{2} & x_{7}\end{array}\right),\left(\begin{array}{llll}x_{5} & y_{2} & x_{6} & y_{9} \\ y_{7} & x_{3} & y_{8} & x_{8}\end{array}\right),\left(\begin{array}{llll}x_{7} & y_{3} & x_{8} & y_{4} \\ y_{1} & x_{6} & y_{2} & x_{5}\end{array}\right),\left(\begin{array}{llll}x_{1} & y_{5} & x_{2} & y_{6} \\ y_{4} & x_{8} & y_{9} & x_{7}\end{array}\right)$,

$\left(\begin{array}{llll}x_{1} & y_{7} & x_{2} & y_{8} \\ y_{9} & x_{3} & y_{3} & x_{4}\end{array}\right)$.

Lemma 2.4. There exists an $L_{8}$ - decomposition of $K_{12,6}$.

Proof. We exhibit the $L_{8}$ - decomposition of $K_{12,6}$ as follows:

$\left(\begin{array}{cccc}x_{1} & y_{1} & x_{2} & y_{2} \\ y_{3} & x_{12} & y_{4} & x_{11}\end{array}\right),\left(\begin{array}{llll}x_{3} & y_{3} & x_{4} & y_{4} \\ y_{5} & x_{2} & y_{6} & x_{1}\end{array}\right),\left(\begin{array}{llll}x_{5} & y_{5} & x_{6} & y_{6} \\ y_{1} & x_{1} & y_{2} & x_{2}\end{array}\right),\left(\begin{array}{llll}x_{7} & y_{1} & x_{8} & y_{2} \\ y_{3} & x_{9} & y_{4} & x_{10}\end{array}\right)$,

$\left(\begin{array}{cccc}x_{9} & y_{3} & x_{10} & y_{4} \\ y_{6} & x_{11} & y_{5} & x_{12}\end{array}\right),\left(\begin{array}{cccc}x_{11} & y_{5} & x_{12} & y_{6} \\ y_{1} & x_{2} & y_{2} & x_{1}\end{array}\right),\left(\begin{array}{cccc}x_{3} & y_{1} & x_{4} & y_{2} \\ y_{6} & x_{10} & y_{5} & x_{9}\end{array}\right),\left(\begin{array}{cccc}x_{5} & y_{3} & x_{6} & y_{4} \\ y_{2} & x_{12} & y_{1} & x_{11}\end{array}\right)$,

$\left(\begin{array}{lllc}x_{7} & y_{5} & x_{8} & y_{6} \\ y_{4} & x_{9} & y_{3} & x_{10}\end{array}\right)$.

Lemma 2.5. There is no $L_{8}$ - decomposition of $K_{8,5}$. 
Proof. Let $A$ and $B$ be the partite set of $K_{8,5}$ such that $|A|=8$ and $|B|=5$. In $L_{8}$, four vertices are of degree 3 and four vertices are of degree 1 . Since $K_{8,5}$ is a bipartite graph, then $L_{8}$ has two vertices of degree 3 and two vertices of degree 1 in one partite set $A$ and similarly in $B$. Total number of edges in $K_{8,5}$ is 40 , then we have $5 L_{8}$ in $K_{8,5}$. First we pull out $4 L_{8}$ from $K_{8,5}$ (as shown in Fig.2). Since each vertices in $A$ has degree 5 , the remaining degree of each vertices of $K_{8,5} \backslash 4 L_{8}$ in the set $A$ is 1 . Here we can't find a $L_{8}$ in $K_{8,5} \backslash 4 L_{8}$, since we need atleast two vertices of degree 3 . Hence we conclude that $L_{8}$-decomposition does not exists in $K_{8,5}$.
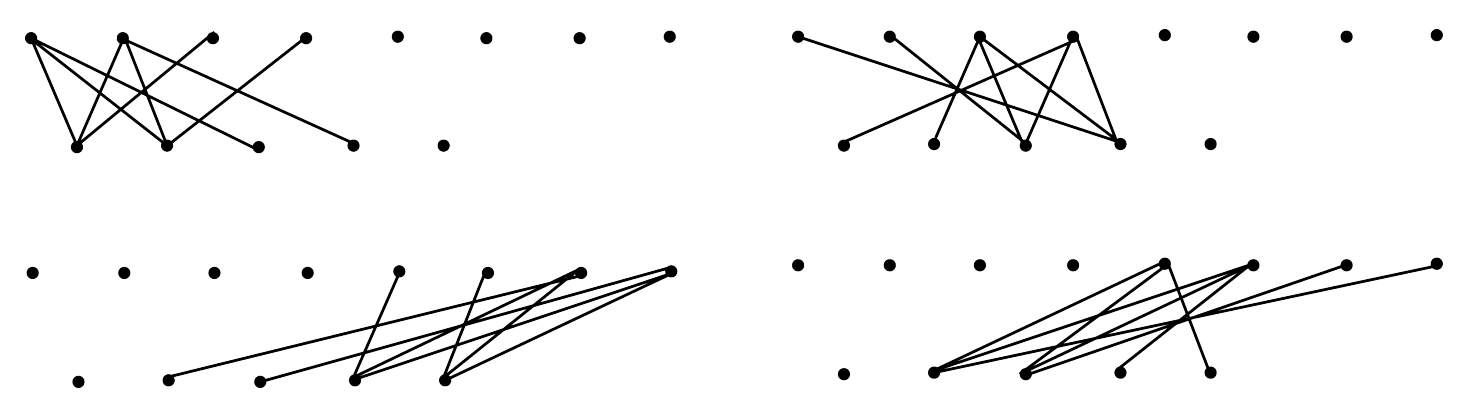

Figure 2. $4 L_{8}$ in $K_{8,5}$

Lemma 2.6. There is no $L_{8}$-decomposition of $K_{4, n}$ for $n \equiv 2(\bmod 4)$.

Proof. Let $n=4 s+2$ for some $s>0$. Suppose that $K_{4, n}$ has an $L_{8}$-decomposition, then it has $(2 s+1) L_{8}$. Let $A=\left\{x_{1}, x_{2}, x_{3}, x_{4}\right\}$ and $B=\left\{y_{1}, y_{2}, \ldots, y_{4 s+1}, y_{4 s+2}\right\}$ be the partite sets of $K_{4, n}$. Consider the $(2 s) L_{8}=\left\{L_{8}^{1}, L_{8}^{2}, \ldots, L_{8}^{2 s}\right\}$ which exists in $K_{4, n-2}$. Now we have to find the last $L_{8}$ i.e., $L_{8}^{2 s+1}$.

Let $\left(x_{1} y_{4 s+1} x_{2} y_{4 s+2}\right)$ be a cycle in $K_{4, n}$. Then join $y_{4 s+1}$ to $x_{3}$ and $y_{4 s+2}$ to $x_{4}$. Now we have to find pendant edges to the vertices $x_{1}$ and $x_{2}$. Suppose there are vertices $y_{a}$ and $y_{b}$ which are joined to $x_{1}$ and $x_{2}$, resp, as the pendant edges in $L_{8}^{t_{1}}$ for some $t_{1} \in\{1,2, \ldots, 2 s\}$. Then we can join these edges to the vertices $x_{1}$ and $x_{2}$ in $L_{8}^{2 s+1}$. Suppose $y_{a}=y_{4 s+1}$ and $y_{b}=y_{4 s+2}$ or viceversa, then we can join the remaining edges $x_{3} y_{4 s+2}, x_{4} y_{4 s+1}$ in $K_{4, n}$ to $y_{a}$ and $y_{b}$, resp. Therefore $\operatorname{deg}_{L_{8}^{t_{1}}}\left(y_{4 s+1}\right)=\operatorname{deg}_{L_{8}^{t_{1}}}\left(y_{4 s+2}\right)=3$ and $\operatorname{deg}_{L_{8}^{2 s+1}}\left(y_{4 s+1}\right)=\operatorname{deg}_{L_{8}^{2 s+1}}\left(y_{4 s+2}\right)=3$. This implies $\left.\operatorname{deg}\left(y_{4 s+1}\right)=\operatorname{deg}_{(} y_{4 s+2}\right)=6$, which is a contradiction. Therefore $y_{a} \neq y_{4 s+1}$ and $y_{b} \neq y_{4 s+2}$ or viceversa.

Then we find the pendant edges to $y_{a}$ and $y_{b}$. There exist vertices $x_{i}$ and $x_{j}, i, j \in\{1,2,3,4\}$ which are joined to $y_{a}$ and $y_{b}$, resp, as the pendant edges in $L_{8}^{t_{2}}$ for some $t_{1} \neq t_{2} \in\{1,2, \ldots, 2 s\}$. Then we can join these edges to the vertices $y_{a}$ and $y_{b}$ in $L_{8}^{t_{1}}$. Now $x_{i} \neq x_{3}$ and $x_{j} \neq x_{4}$ or viceversa, since $x_{3} y_{a}, x_{3} y_{b}, x_{4} y_{a}$ and $x_{4} y_{b}$ are the edges of the cycle in $L_{8}^{t_{1}}$. Then $x_{i}, x_{j}$ must be $x_{1}, x_{2}$. Again we have to find the pendant edges to $x_{1}, x_{2}$. Repeat the above procedure cyclically we get to find the pendant edges of $x_{1}, x_{2}$. Therefore we can't find the pendant edges to $x_{1}, x_{2}$. Hence the proof.

Theorem 2.7. For any $m, n \geq 4, K_{m, n}$ has an $L_{8}$-decomposition if and only if $m n \equiv 0(\bmod 8)$ except $(m, n)=(4,2(\bmod 4)) \&(8,5)$.

Proof. Necessity. We first observe that $K_{m, n}$ has $m+n$ vertices and $m n$ edges. Assume that $K_{m, n}$ admits an $L_{8}$ - decomposition. Then the number of edges in the graph must be divisible by 8 i.e., $8 \mid m n$ and hence $m n \equiv 0(\bmod 8)$. Further, $(m, n) \neq(4,2(\bmod 4)) \&(8,5)$ follows from Lemmas 2.6 and 2.5 .

Sufficiency. We construct the required decomposition in two cases.

Case $(1) m($ or $) n \equiv 0(\bmod 8)$.

Suppose we take $m \equiv 0(\bmod 8)$. Further we divide the proof into four subcases.

Subcase $(i) m \equiv 0(\bmod 8)$ and $n \equiv 0(\bmod 4)$.

Let $m=8 s$ and $n=4 t$ for some $s, t>0$. Then we can write $K_{m, n}=2 s t K_{4,4}$. We know that $K_{4,4}$ has an $L_{8}$-decomposition(see Fig.2). 
Subcase $($ ii) $m \equiv 0(\bmod 8)$ and $n \equiv 1(\bmod 4)$.

Let $m=8 s$ and $n=4 t+1$ for some $s, t>1$, since for $s=t=1, K_{m, n}$ has no $L_{8}$ - decomposition by Lemma 2.5. Then we can write $K_{m, n}=2 s(t-2) K_{4,4} \oplus s K_{8,9}$. Then by Lemma 2.3, we get an $L_{8}$ decomposition of $K_{m, n}$.

Subcase $($ iii $) m \equiv 0(\bmod 8)$ and $n \equiv 2(\bmod 4)$.

Let $m=8 s$ and $n=4 t+2$ for some $s, t>0$. Then we can write $K_{m, n}=2 s(t-1) K_{4,4} \oplus s K_{8,6}$. Then by Lemma 2.1, we get an $L_{8}$ - decomposition of $K_{m, n}$.

Subcase $(i v) m \equiv 0(\bmod 8)$ and $n \equiv 3(\bmod 4)$.

Let $m=8 s$ and $n=4 t+3$ for some $s, t>0$. Then we can write $K_{m, n}=2 s(t-1) K_{4,4} \oplus s K_{8,7}$. Then by Lemma 2.2, we get an $L_{8}$ - decomposition of $K_{m, n}$.

Case $(2) m \equiv 0(\bmod 4)$ and $n \equiv 0(\bmod 2)$.

Subcase $(i) m \equiv 0(\bmod 4)$ and $n \equiv 0(\bmod 4)$.

Let $m=4 s$ and $n=4 t$ for some $s, t>0$. Then we can write $K_{m, n}=s t K_{4,4}$. We know that $K_{4,4}$ has an $L_{8}$-decomposition.

Subcase $($ ii $) m \equiv 0(\bmod 4)$ and $n \equiv 2(\bmod 4)$.

Let $m=4 s$ and $n=4 t+2$ for some $s, t>0$. For $s=1, K_{4, n}$ has no $L_{8}$ - decomposition by Lemma 2.6. Consider $s \geq 2$. For even $s, m$ must be the multiple of 8 . Then by case(1), result is proved for even $s$. It is sufficient to prove the case for odd $s$. Consider $s$ is odd and $s \geq 3$. Then we can write $K_{m, n}=s(t-1) K_{4,4} \oplus K_{4(s-3), 6} \oplus K_{12,6}$. Since $s$ is odd, $s-3$ is even. Hence the results follows by the above cases and by the Lemma 2.4 .

\section{3. $\quad L_{8}$ - decomposition of $K_{m} \times K_{n}$}

In this section we investigate the existence of $L_{8}$ - decomposition of the tensor product of complete graphs.

Lemma 3.1. For an even integer $k>2$ and any graph $G$, there exists an $L_{2 k}$-decomposition of $L_{2 k} \times G$.

Proof. Let $L_{2 k}$ be $\left(\begin{array}{cccc}x_{1} & x_{2} & \ldots & x_{k} \\ x_{k+1} & x_{k+2} & \ldots & x_{2 k}\end{array}\right)$ and $y_{j_{1}} y_{j_{2}}$ be any edge in $G$, then the induced subgraph $\left\langle L_{2 k} \times\left\{y_{j_{1}} y_{j_{2}}\right\}\right\rangle$ of $L_{2 k} \times G$ gives two $L_{2 k}$ 's as follows:

$\left(\begin{array}{ccccc}x_{1}^{j_{1}} & x_{2}^{j_{2}} & x_{3}^{j_{1}} & \ldots & x_{k}^{j_{2}} \\ x_{k+1}^{j_{2}} & x_{k+2}^{j_{1}} & x_{k+3}^{j_{2}} & \ldots & x_{2 k}^{j_{1}}\end{array}\right),\left(\begin{array}{ccccc}x_{1}^{j_{2}} & x_{2}^{j_{1}} & x_{3}^{j_{2}} & \ldots & x_{k}^{j_{1}} \\ x_{k+1}^{j_{1}} & x_{k+2}^{j_{2}} & x_{k+3}^{j_{1}} & \ldots & x_{2 k}^{j_{2}}\end{array}\right)$. So, for each edge in $G$ there are two $L_{2 k}$ 's in $L_{2 k} \times G$, and hence we have $2|E(G)| L_{2 k}$ 's in $L_{2 k} \times G$.

Lemma 3.2. There exists an $L_{8}-$ decomposition of $K_{4} \times K_{4}$.

Proof. The $L_{8}$ - decomposition of $K_{4} \times K_{4}$ is given below.

$\left(\begin{array}{llll}x_{1}^{j_{1}} & x_{2}^{j_{2}} & x_{3}^{j_{1}} & x_{4}^{j_{2}} \\ x_{3}^{j_{2}} & x_{4}^{j_{1}} & x_{1}^{j_{2}} & x_{2}^{j_{1}}\end{array}\right)$ for $j_{1}<j_{2} \in\{1,2,3,4\},\left(\begin{array}{cccc}x_{1}^{j+2} & x_{2}^{j} & x_{3}^{j+2} & x_{4}^{j} \\ x_{4}^{j+1} & x_{1}^{j+1} & x_{2}^{j+1} & x_{3}^{j+1}\end{array}\right)$ for $j=1,2,\left(\begin{array}{llll}x_{1}^{4} & x_{2}^{1} & x_{3}^{4} & x_{4}^{1} \\ x_{2}^{3} & x_{3}^{2} & x_{4}^{3} & x_{1}^{2}\end{array}\right)$.

Lemma 3.3. There exists an $L_{8}$ - decomposition of $K_{4} \times K_{5}$.

Proof. The $L_{8}$ - decomposition of $K_{4} \times K_{5}$ is given as follows:

$\left(\begin{array}{cccc}x_{1}^{j_{1}} & x_{2}^{j_{2}} & x_{3}^{j_{1}} & x_{4}^{j_{2}} \\ x_{3}^{j_{2}} & x_{4}^{j_{1}} & x_{1}^{j_{2}} & x_{2}^{j_{1}}\end{array}\right)$ for $j_{1}<j_{2} \in\{1,2,3,4,5\}$ except $\left(j_{1}, j_{2}\right)=(2,4),(3,4)$

$\left(\begin{array}{cccc}x_{1}^{3} & x_{2}^{4} & x_{3}^{3} & x_{4}^{4} \\ x_{3}^{4} & x_{1}^{5} & x_{1}^{4} & x_{3}^{5}\end{array}\right),\left(\begin{array}{llll}x_{1}^{2} & x_{2}^{4} & x_{3}^{2} & x_{4}^{4} \\ x_{3}^{4} & x_{3}^{5} & x_{1}^{4} & x_{1}^{5}\end{array}\right),\left(\begin{array}{cccc}x_{1}^{3} & x_{2}^{1} & x_{3}^{3} & x_{4}^{1} \\ x_{2}^{2} & x_{1}^{2} & x_{4}^{2} & x_{3}^{2}\end{array}\right),\left(\begin{array}{llll}x_{1}^{4} & x_{2}^{1} & x_{3}^{4} & x_{4}^{1} \\ x_{2}^{3} & x_{3}^{2} & x_{4}^{3} & x_{1}^{2}\end{array}\right)$,

$\left(\begin{array}{llll}x_{1}^{4} & x_{2}^{2} & x_{3}^{4} & x_{4}^{2} \\ x_{4}^{3} & x_{3}^{3} & x_{2}^{3} & x_{1}^{3}\end{array}\right),\left(\begin{array}{llll}x_{1}^{5} & x_{2}^{2} & x_{3}^{5} & x_{4}^{2} \\ x_{2}^{1} & x_{4}^{4} & x_{4}^{1} & x_{2}^{4}\end{array}\right),\left(\begin{array}{llll}x_{1}^{5} & x_{2}^{3} & x_{3}^{5} & x_{4}^{3} \\ x_{4}^{1} & x_{4}^{4} & x_{2}^{1} & x_{2}^{4}\end{array}\right)$.

Lemma 3.4. There exists an $L_{8}$ - decomposition of $K_{4} \times K_{4,5}$. 
Proof. We can write $K_{4,5}=K_{4,4} \oplus K_{4,1}$. Now $K_{4} \times K_{4,5}=\left(K_{4} \times K_{4,4}\right) \oplus\left(K_{4} \times K_{4,1}\right)$. By Theorem 2.7 and Lemma 3.1, it is sufficient to prove the existence of $L_{8}$ - decomposition of $K_{4} \times K_{4,1}$. The $L_{8}$-decomposition of $K_{4} \times K_{4,1}$ shown in Fig.3 gives the required decomposition.
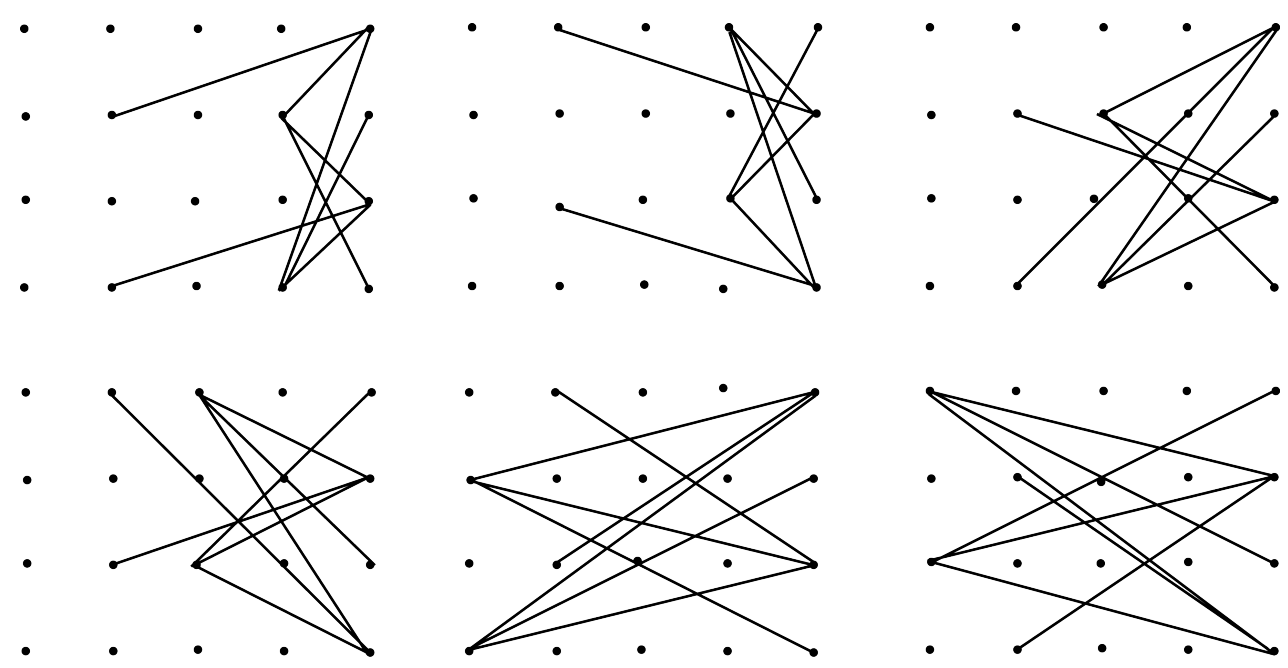

Figure 3. $L_{8}$ decomposition of $K_{4} \times K_{4,1}$.

Lemma 3.5. There exists an $L_{8}$ - decomposition of $K_{8} \times K_{7}$.

Proof. We can write $K_{8}=3 L_{8} \oplus C_{4}$ (see Fig.4). Now $K_{8} \times K_{7}=3\left(L_{8} \times K_{7}\right) \oplus\left(C_{4} \times K_{7}\right)$. To complete the proof, by Lemma 3.1, it is sufficient to prove the existence of $L_{8}$ - decomposition of $C_{4} \times K_{7}$, which is given as follows:

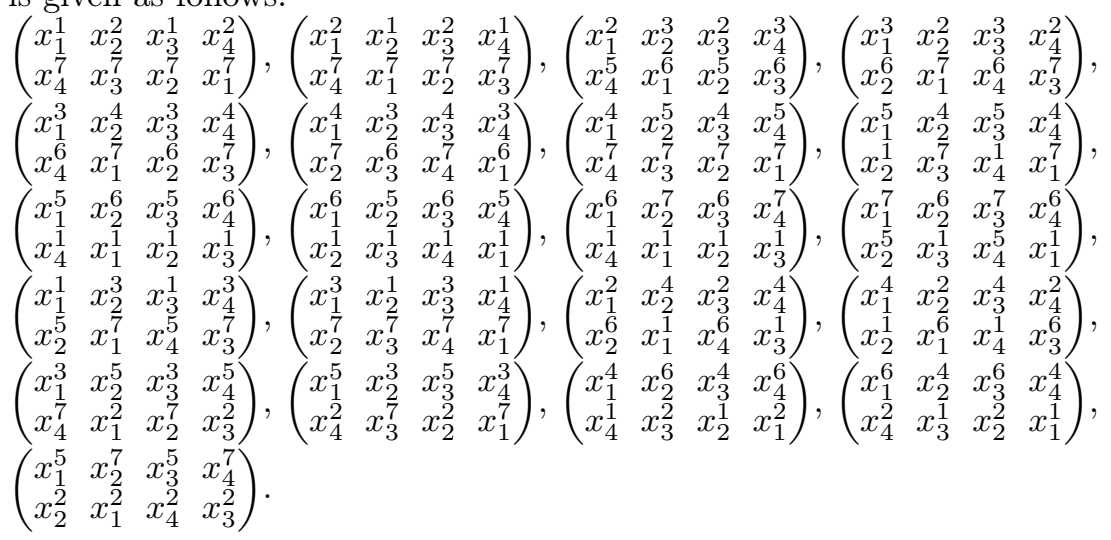
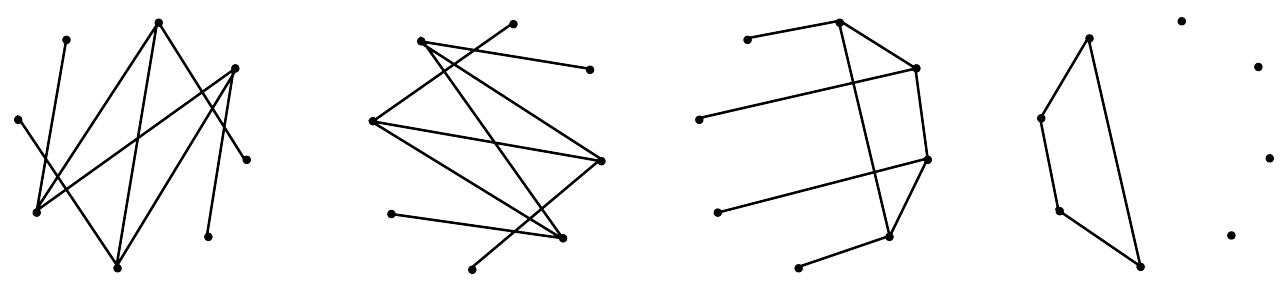

Figure 4. $K_{8}=3 L_{8} \oplus C_{4}$.

Lemma 3.6. There exists an $L_{8}$-decomposition of $K_{8} \times K_{4,7}$. 
Proof. We can write $K_{8} \times K_{4,7}=2\left(K_{4} \times K_{4,4}\right) \oplus 2\left(K_{4} \times K_{4,3}\right) \oplus\left(K_{4,4} \times K_{4,4}\right) \oplus\left(K_{4,4} \times K_{4,3}\right)$. By Theorem 2.7 and Lemma 3.1, it is sufficient to prove the existence of $L_{8^{-}}$decomposition of $K_{4} \times K_{4,3}$. The $L_{8}$ - decomposition of $K_{4} \times K_{4,3}$ is given below.

$$
\begin{aligned}
& \left(\begin{array}{cccc}
x_{1}^{j_{1}} & x_{2}^{j_{2}} & x_{3}^{1} & x_{4}^{j_{2}} \\
x_{3}^{j_{2}} & x_{4}^{j_{1}+1} & x_{1}^{j_{2}} & x_{2}^{j_{1}+1}
\end{array}\right),\left(\begin{array}{cccc}
x_{1}^{j_{2}} & x_{2}^{j_{1}} & x_{3}^{j_{2}} & x_{4}^{j_{1}} \\
x_{3}^{j_{1}+1} & x_{4}^{j_{2}} & x_{1}^{j_{1}+1} & x_{2}^{j_{2}}
\end{array}\right) \text { for } j_{1}=3, j_{2} \in\{5,6,7\} \\
& \left(\begin{array}{cccc}
x_{1}^{j_{1}} & x_{2}^{j_{2}} & x_{3}^{j_{1}} & x_{4}^{j_{2}} \\
x_{3}^{j_{2}} & x_{3}^{j_{1}+2} & x_{1}^{j_{2}} & x_{1}^{j_{1}+2}
\end{array}\right),\left(\begin{array}{cccc}
x_{1}^{j_{2}} & x_{2}^{j_{1}} & x_{3}^{j_{2}} & x_{4}^{j_{1}} \\
x_{4}^{j_{1}+2} & x_{4}^{j_{2}} & x_{2}^{j_{1}+2} & x_{2}^{j_{2}}
\end{array}\right) \text { for } j_{1}=2, j_{2} \in\{5,6,7\} \\
& \left(\begin{array}{lccc}
x_{1}^{j_{1}} & x_{2}^{j_{2}} & x_{3}^{j_{1}} & x_{4}^{j_{2}} \\
x_{3}^{j_{2}} & x_{1}^{j_{1}+3} & x_{1}^{j_{2}} & x_{3}^{j_{1}+3}
\end{array}\right),\left(\begin{array}{cccc}
x_{1}^{j_{2}} & x_{2}^{j_{1}} & x_{3}^{j_{2}} & x_{4}^{j_{1}} \\
x_{2}^{j_{1}+3} & x_{4}^{j_{2}} & x_{4}^{j_{1}+3} & x_{2}^{j_{2}}
\end{array}\right) \text { for } j_{1}=1, j_{2} \in\{5,6,7\} .
\end{aligned}
$$

Lemma 3.7. There exists an $L_{8}$-decomposition of $K_{5} \times K_{5}$.

Proof. We exhibit the $L_{8}$ - decomposition of $K_{5} \times K_{5}$ as follows:

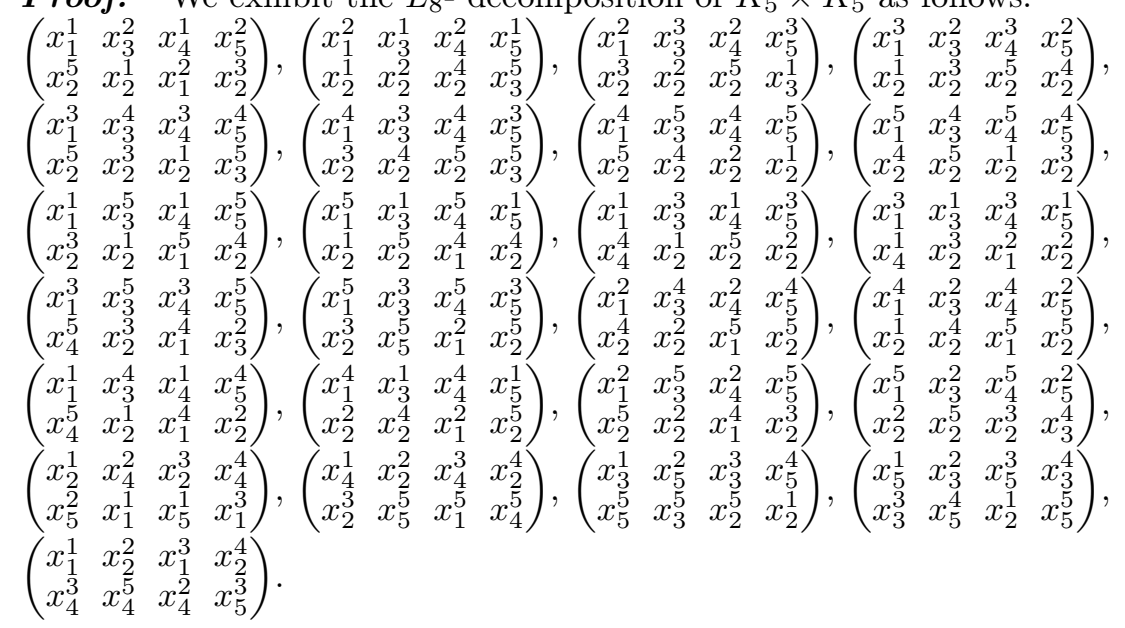

Theorem 3.8. $K_{m} \times K_{n}$ has an $L_{8}$ - decomposition if and only if $m n(m-1)(n-1) \equiv 0(\bmod 16)$.

Proof. Necessity. Assume that $K_{m} \times K_{n}$ admits an $L_{8^{-}}$decomposition. Then the number of edges in the graph $K_{m} \times K_{n}$ is $\frac{m n(m-1)(n-1)}{2}$ which should be divisible by 8 , the number of edges in $L_{8}$ i.e., $16 \mid m n(m-1)(n-1)$ and hence $m n(m-1)(n-1) \equiv 0(\bmod 16)$.

Sufficiency. We construct the required decomposition in the following cases.

Case $(1) m, n \equiv 0(\bmod 4)$.

Let $m=4 s$ and $n=4 t$ for some $s, t>0$. Then we can write $K_{m} \times K_{n}=s t\left(K_{4} \times K_{4}\right) \oplus$ $2 s t(s-1)(t-1) K_{4,16} \oplus 2 s t(s+t-2) K_{4,12}$. By Lemma 3.2 and Theorem 2.7 the graph $K_{m} \times K_{n}$ has the desired decomposition.

Case $(2) m \equiv 0(\bmod 4), n \equiv 1(\bmod 4)$.

Let $m=4 s$ and $n=4 t+1$ for some $s, t>0$. Then we can write $K_{m} \times K_{n}=\left(K_{4 s} \times K_{4(t-1)}\right) \oplus$ $s\left(K_{4} \times K_{5}\right) \oplus\left(\frac{5 s(s-1)}{2}\right) K_{4,16} \oplus s(t-1)\left(K_{4} \times K_{4,5}\right) \oplus 4 s(s-1)(t-1) K_{4,20}$. By the Case (1) above, Lemmas 3.3, 3.4 and Theorem 2.7 the graph $K_{m} \times K_{n}$ has the desired decomposition.

Case $(3) m \equiv 0(\bmod 8), n \equiv 3(\bmod 4)$.

Subcase $(i) m=8$ and $n \equiv 3(\bmod 4)$

Let $n=4 t+3$ for some $t>0$. Then we can write $K_{8} \times K_{n}=\left(K_{8} \times K_{4(t-1)}\right) \oplus\left(K_{8} \times K_{7}\right) \oplus$ $(t-1)\left(K_{8} \times K_{4,7}\right)$. The $L_{8}$ - decomposition of all the three terms follows from Case (1) and the Lemmas $3.5,3.6$.

Subcase $($ ii) $m \equiv 0(\bmod 8), m>8$ and $n \equiv 3(\bmod 4)$

Now, let $m=8 s$ for some $s>1$. Then we can write $K_{m} \times K_{n}=s\left(K_{8} \times K_{n}\right) \oplus\left(\frac{s(s-1)}{2}\right)\left(K_{8,8} \times K_{n}\right)$. $K_{m} \times K_{n}$ has the desired decomposition, by the Theorem 2.7, Lemma 3.1 and Subcase 3(i) above. Case $(4) m \equiv 0(\bmod 16)$. 
Let $m=16 s$, for some $s>0$. We can write $K_{m} \times K_{n}=s\left(K_{16} \times K_{n}\right) \oplus\left(\frac{s(s-1)}{2}\right)\left(K_{16,16} \times K_{n}\right)$. The $L_{8^{-}}$decomposition of the terms in RHS follows from Lemma 3.1 and Theorems 1.3, 2.7 .

Case $(5) m \equiv 1(\bmod 16)$.

Let $m=16 s+1$, for some $s>0$. Then by Theorem 1.3, we have $L_{8}$ - decomposition of $K_{16 s+1}$, for any $s>0$. Then by Lemma $3.1, K_{m} \times K_{n}$ has an $L_{8^{-}}$decomposition.

Case $(6) m \equiv 1(\bmod 4), n \equiv 1(\bmod 4)$.

Let $m=4 s+1$ and $n=4 t+1$ for some $s, t>0$. Then we can write $K_{m} \times K_{n}=\left(K_{4(s-1)} \times K_{4(t-1)}\right)$ $\oplus\left(K_{4(s-1)} \times K_{5}\right) \oplus\left(K_{5} \times K_{4(t-1)}\right) \oplus 2(s-1)(t-1)\left(K_{4} \times K_{4,5}\right) \oplus 4(s-1)(t-1)(s+t-4) K_{4,20} \oplus$ $K_{5} \times K_{5} \oplus 5(s+t-2) K_{4,20} \oplus 2(s-1)(t-1) K_{16,25}$. Then by the Cases (1), (2) above and by Lemmas 3.4, 3.7 and Theorem 2.7, the graph $K_{m} \times K_{n}$ has the desired decomposition.

\section{4. $L_{8}$ - decomposition of $K_{m} \otimes \overline{K_{n}}$}

In this section we investigate the existence of $L_{8^{-}}$decomposition of wreath product of complete graphs.

Lemma 4.1. If the graph $G$ has an $L_{2 k}$-decomposition, then $G \otimes \overline{K_{n}}$ has an $L_{2 k}$-decomposition for any $n>0$ and even $k>2$.

Proof. Let $G$ has an $L_{2 k^{-}}$decomposition. For each $L_{2 k},\left(\begin{array}{cccc}x_{1} & x_{2} & \ldots & x_{k} \\ x_{k+1} & x_{k+2} & \ldots & x_{2 k}\end{array}\right)$, in $G$, we exhibit the $L_{2 k^{-}}$decomposition of $L_{2 k} \otimes \overline{K_{n}}$ as follows: $\left(\begin{array}{cccc}x_{1}^{j_{1}} & x_{2}^{j_{2}} & \ldots & x_{k}^{j_{2}} \\ x_{k+1}^{j_{2}} & x_{k+2}^{j_{1}} & \ldots & x_{2 k}^{j_{1}}\end{array}\right)$ for $j_{1} \leq j_{2} \in\{1,2, \ldots, n\}$, $\left(\begin{array}{cccc}x_{1}^{j_{2}} & x_{2}^{j_{1}} & \ldots & x_{k}^{j_{1}} \\ x_{k+1}^{j_{1}} & x_{k+2}^{j_{2}} & \ldots & x_{2 k}^{j_{2}}\end{array}\right)$ for $j_{1}<j_{2} \in\{1,2, \ldots, n\}$.

Lemma 4.2. There exists an $L_{8}$ - decomposition of $K_{4,5} \otimes \overline{K_{6}}$.

Proof. The $L_{8}$ - decomposition of $K_{4,5} \otimes \overline{K_{6}}$ is given as follows:

$\left(\begin{array}{cccc}x_{1}^{j_{1}} & x_{5}^{j_{2}} & x_{2}^{j_{1}} & x_{6}^{j_{2}} \\ x_{7}^{j_{2}} & x_{3}^{j_{1}} & x_{8}^{j_{2}} & x_{4}^{j_{1}}\end{array}\right)$ for $j_{1} \leq j_{2} \in\{1,2,3,4,5,6\}$ except $\left(j_{1}, j_{2}\right)=(4,4),(2,3)$,

$(2,4),(3,4)$,

$\left(\begin{array}{cccc}x_{1}^{j_{2}} & x_{5}^{j_{1}} & x_{2}^{j_{2}} & x_{6}^{j_{1}} \\ x_{7}^{j_{1}} & x_{3}^{j_{2}} & x_{8}^{j_{1}} & x_{4}^{j_{2}}\end{array}\right)$ for $j_{1}<j_{2} \in\{1,2,3,4,5,6\}$ except $\left(j_{1}, j_{2}\right)=(3,4)$

$(3,5),(3,6),(4,5),(4,6)$,

$\left(\begin{array}{cccc}x_{3}^{j_{1}} & x_{7}^{j_{2}} & x_{4}^{j_{1}} & x_{8}^{j_{2}} \\ x_{6}^{j_{2}} & x_{2}^{j_{1}} & x_{5}^{j_{2}} & x_{1}^{j_{1}}\end{array}\right)$ for $j_{1} \leq j_{2} \in\{1,2,3,4,5,6\}$ except $\left(j_{1}, j_{2}\right)=(2,4),(2,5)$,

$\left(\begin{array}{cccc}x_{3}^{j_{2}} & x_{7}^{j_{1}} & x_{4}^{j_{2}} & x_{8}^{j_{1}} \\ x_{6}^{j_{1}} & x_{2}^{j_{2}} & x_{5}^{j_{1}} & x_{1}^{j_{2}}\end{array}\right)$ for $j_{1}<j_{2} \in\{1,2,3,4,5,6\}$ except $\left(j_{1}, j_{2}\right)=(3,5),(4,5)$,

$\left(\begin{array}{llll}x_{i_{1}}^{2} & x_{i_{2}}^{1} & x_{i_{1}}^{4} & x_{i_{2}}^{3} \\ x_{i_{2}}^{5} & x_{i_{1}}^{3} & x_{i_{2}}^{4} & x_{i_{1}}^{1}\end{array}\right),\left(\begin{array}{llll}x_{i_{1}}^{1} & x_{i_{2}}^{2} & x_{i_{1}}^{3} & x_{i_{2}}^{4} \\ x_{i_{2}}^{5} & x_{i_{1}}^{6} & x_{i_{2}}^{3} & x_{i_{1}}^{2}\end{array}\right),\left(\begin{array}{cccc}x_{i_{1}}^{4} & x_{i_{2}}^{2} & x_{i_{1}}^{5} & x_{i_{2}}^{6} \\ x_{i_{2}}^{5} & x_{i_{1}}^{2} & x_{i_{2}}^{3} & x_{i_{1}}^{6}\end{array}\right)$ for $i_{1} \in\{1,2\} \& i_{2} \in\{9\}$,

$\left(\begin{array}{llll}x_{i_{1}}^{5} & x_{i_{2}}^{1} & x_{i_{1}}^{6} & x_{i_{2}}^{5} \\ x_{i_{2}}^{4} & x_{i_{1}}^{1} & x_{i_{2}}^{3} & x_{i_{1}}^{3}\end{array}\right)$ for $i_{1} \in\{1,2,3,4\} \& i_{2} \in\{9\}$,

$\left(\begin{array}{llll}x_{1}^{4} & x_{5}^{4} & x_{2}^{4} & x_{6}^{4} \\ x_{7}^{4} & x_{3}^{4} & x_{8}^{4} & x_{3}^{5}\end{array}\right),\left(\begin{array}{llll}x_{1}^{2} & x_{9}^{6} & x_{2}^{2} & x_{6}^{3} \\ x_{7}^{3} & x_{2}^{1} & x_{8}^{3} & x_{4}^{2}\end{array}\right),\left(\begin{array}{llll}x_{1}^{2} & x_{5}^{4} & x_{2}^{2} & x_{6}^{4} \\ x_{7}^{4} & x_{3}^{2} & x_{8}^{4} & x_{4}^{3}\end{array}\right),\left(\begin{array}{llll}x_{1}^{3} & x_{5}^{4} & x_{2}^{3} & x_{9}^{6} \\ x_{7}^{4} & x_{3}^{3} & x_{8}^{4} & x_{1}^{1}\end{array}\right)$,

$\left(\begin{array}{llll}x_{1}^{4} & x_{5}^{3} & x_{2}^{4} & x_{6}^{3} \\ x_{7}^{3} & x_{1}^{2} & x_{8}^{3} & x_{4}^{4}\end{array}\right),\left(\begin{array}{llll}x_{1}^{5} & x_{5}^{3} & x_{2}^{5} & x_{6}^{3} \\ x_{7}^{3} & x_{2}^{2} & x_{8}^{3} & x_{4}^{5}\end{array}\right),\left(\begin{array}{llll}x_{1}^{6} & x_{5}^{3} & x_{2}^{6} & x_{6}^{3} \\ x_{7}^{3} & x_{4}^{5} & x_{8}^{3} & x_{4}^{6}\end{array}\right),\left(\begin{array}{cccc}x_{1}^{5} & x_{5}^{4} & x_{2}^{5} & x_{6}^{4} \\ x_{7}^{4} & x_{3}^{5} & x_{8}^{4} & x_{1}^{3}\end{array}\right)$,

$\left(\begin{array}{llll}x_{1}^{6} & x_{5}^{4} & x_{2}^{6} & x_{6}^{4} \\ x_{7}^{4} & x_{3}^{6} & x_{8}^{4} & x_{2}^{3}\end{array}\right),\left(\begin{array}{llll}x_{3}^{2} & x_{7}^{4} & x_{4}^{2} & x_{8}^{4} \\ x_{5}^{3} & x_{2}^{2} & x_{5}^{4} & x_{1}^{2}\end{array}\right),\left(\begin{array}{llll}x_{3}^{2} & x_{7}^{5} & x_{4}^{2} & x_{8}^{5} \\ x_{6}^{5} & x_{2}^{2} & x_{6}^{4} & x_{1}^{2}\end{array}\right),\left(\begin{array}{llll}x_{3}^{5} & x_{7}^{3} & x_{4}^{5} & x_{8}^{3} \\ x_{6}^{3} & x_{2}^{5} & x_{6}^{4} & x_{1}^{5}\end{array}\right)$, 
$\left(\begin{array}{cccc}x_{3}^{5} & x_{7}^{4} & x_{4}^{5} & x_{8}^{4} \\ x_{5}^{3} & x_{2}^{5} & x_{5}^{4} & x_{1}^{5}\end{array}\right),\left(\begin{array}{llll}x_{3}^{2} & x_{9}^{1} & x_{3}^{4} & x_{9}^{3} \\ x_{9}^{5} & x_{3}^{3} & x_{5}^{3} & x_{3}^{1}\end{array}\right),\left(\begin{array}{cccc}x_{4}^{2} & x_{9}^{1} & x_{4}^{4} & x_{9}^{3} \\ x_{9}^{5} & x_{4}^{3} & x_{6}^{4} & x_{4}^{1}\end{array}\right),\left(\begin{array}{cccc}x_{3}^{1} & x_{9}^{2} & x_{3}^{3} & x_{9}^{4} \\ x_{9}^{5} & x_{3}^{6} & x_{9}^{3} & x_{1}^{6}\end{array}\right)$,

$\left(\begin{array}{cccc}x_{4}^{1} & x_{9}^{2} & x_{4}^{3} & x_{9}^{4} \\ x_{9}^{5} & x_{4}^{6} & x_{9}^{3} & x_{2}^{6}\end{array}\right),\left(\begin{array}{llll}x_{3}^{4} & x_{9}^{2} & x_{3}^{5} & x_{9}^{6} \\ x_{9}^{5} & x_{3}^{2} & x_{9}^{3} & x_{3}^{3}\end{array}\right),\left(\begin{array}{llll}x_{4}^{4} & x_{9}^{2} & x_{4}^{5} & x_{9}^{6} \\ x_{9}^{5} & x_{4}^{2} & x_{9}^{3} & x_{4}^{3}\end{array}\right),\left(\begin{array}{llll}x_{3}^{2} & x_{9}^{4} & x_{3}^{6} & x_{9}^{6} \\ x_{6}^{4} & x_{3}^{4} & x_{5}^{3} & x_{3}^{1}\end{array}\right)$,

$\left(\begin{array}{llll}x_{4}^{2} & x_{9}^{4} & x_{4}^{6} & x_{9}^{6} \\ x_{5}^{5} & x_{4}^{4} & x_{6}^{4} & x_{4}^{1}\end{array}\right)$.

Lemma 4.3. There exists an $L_{8}$-decomposition of $K_{4,5} \otimes \overline{K_{10}}$.

Proof. We exhibit the $L_{8^{-}}$decomposition of $K_{4,5} \otimes \overline{K_{10}}$ as follows:

$\left(\begin{array}{llll}x_{i_{1}}^{1} & x_{i_{2}}^{1} & x_{i_{1}}^{2} & x_{i_{2}}^{2} \\ x_{i_{2}}^{7} & x_{i_{1}}^{5} & x_{i_{2}}^{8} & x_{i_{1}}^{6}\end{array}\right),\left(\begin{array}{llll}x_{i_{1}}^{1} & x_{i_{2}}^{3} & x_{i_{1}}^{2} & x_{i_{2}}^{4} \\ x_{i_{2}}^{8} & x_{i_{1}}^{6} & x_{i_{2}}^{7} & x_{i_{1}}^{5}\end{array}\right)$, for $i_{1} \in\{1,2,3,4\} \& i_{2} \in\{5,6,7,8,9\}$

$\left(\begin{array}{cccc}x_{i_{1}}^{3} & x_{i_{2}}^{1} & x_{i_{1}}^{4} & x_{i_{2}}^{2} \\ x_{i_{2}}^{9} & x_{i_{1}}^{7} & x_{i_{2}}^{10} & x_{i_{1}}^{8}\end{array}\right),\left(\begin{array}{cccc}x_{i_{1}}^{1} & x_{i_{2}}^{5} & x_{i_{1}}^{2} & x_{i_{2}}^{6} \\ x_{i_{2}}^{9} & x_{i_{1}}^{9} & x_{i_{2}}^{10} & x_{i_{1}}^{10}\end{array}\right)$ for $i_{1} \in\{1,2,3,4\} \& i_{2} \in\{5,6,7,8,9\}$,

$\left(\begin{array}{llll}x_{i_{1}}^{3} & x_{i_{2}}^{5} & x_{i_{1}}^{4} & x_{i_{2}}^{6} \\ x_{i_{2}}^{8} & x_{i_{1}}^{8} & x_{i_{2}}^{7} & x_{i_{1}}^{7}\end{array}\right)$ for $i_{1} \in\{1,2,3,4\} \& i_{2} \in\{5,6,7,8,9\}$,

$\left(\begin{array}{cccc}x_{i_{1}}^{9} & x_{i_{2}}^{1} & x_{i_{1}}^{10} & x_{i_{2}}^{2} \\ x_{i_{2}}^{3} & x_{i_{1}}^{6} & x_{i_{2}}^{4} & x_{i_{1}}^{5}\end{array}\right)$ for $i_{1} \in\{1,2,3,4\} \& i_{2} \in\{5,6,7,8,9\}$ except $\left(i_{1}, i_{2}\right)=(1,9)$

$\left(\begin{array}{cccc}x_{i_{1}}^{3} & x_{i_{2}}^{3} & x_{i_{1}}^{4} & x_{i_{2}}^{4} \\ x_{i_{2}}^{7} & x_{i_{1}}^{5} & x_{i_{2}}^{8} & x_{i_{1}}^{6}\end{array}\right)$ for $i_{1} \in\{1,2,3,4\} \& i_{2} \in\{5,6,7,8,9\}$ except $\left(i_{1}, i_{2}\right)=(3,7),(4,8)$

$\left(\begin{array}{cccc}x_{i_{1}}^{5} & x_{i_{2}}^{5} & x_{i_{1}}^{6} & x_{i_{2}}^{6} \\ x_{i_{2}}^{9} & x_{i_{1}}^{7} & x_{i_{2}}^{10} & x_{i_{1}}^{8}\end{array}\right)$ for $i_{1} \in\{1,2,3,4\} \& i_{2} \in\{5,6,7,8,9\}$ except $\left(i_{1}, i_{2}\right)=(3,5),(4,6)$

$\left(\begin{array}{cccc}x_{i_{1}}^{7} & x_{i_{2}}^{7} & x_{i_{1}}^{8} & x_{i_{2}}^{8} \\ x_{i_{2}}^{2} & x_{i_{1}}^{9} & x_{i_{2}}^{1} & x_{i_{1}}^{10}\end{array}\right)$ for $i_{1} \in\{1,2,3,4\} \& i_{2} \in\{5,6,7,8,9\}$ except $\left(i_{1}, i_{2}\right)=(3,7),(4,8)$

$\left(\begin{array}{llll}x_{i_{1}}^{9} & x_{i_{2}}^{9} & x_{i_{1}}^{10} & x_{i_{2}}^{10} \\ x_{i_{2}}^{6} & x_{i_{1}}^{2} & x_{i_{2}}^{5} & x_{i_{1}}^{1}\end{array}\right)$ for $i_{1} \in\{1,2,3,4\} \& i_{2} \in\{5,6,7,8,9\}$ except $\left(i_{1}, i_{2}\right)=(1,5),(1,6)$

$\left(\begin{array}{cccc}x_{i}^{5} & x_{i_{2}}^{7} & x_{i_{1}}^{6} & x_{i_{2}}^{8} \\ x_{i_{2}}^{10} & x_{i_{1}}^{10} & x_{i_{2}}^{9} & x_{i_{1}}^{9}\end{array}\right)$ for $i_{1} \in\{1,2,3,4\} \& i_{2} \in\{5,6,7,8,9\}$ except $\left(i_{1}, i_{2}\right)=(3,7),(4,8)$

$\left(\begin{array}{llll}x_{i_{1}}^{7} & x_{i_{2}}^{9} & x_{i_{1}}^{8} & x_{i_{2}}^{10} \\ x_{i_{2}}^{3} & x_{i_{1}}^{4} & x_{i_{2}}^{4} & x_{i_{1}}^{3}\end{array}\right)$ for $i_{1} \in\{1,2,3,4\} \& i_{2} \in\{5,6,7,8,9\}$ except $\left(i_{1}, i_{2}\right)=(1,9),(3,7)$,

$(4,8)$

$\left(\begin{array}{cccc}x_{1}^{9} & x_{9}^{1} & x_{1}^{10} & x_{9}^{2} \\ x_{6}^{4} & x_{1}^{6} & x_{5}^{4} & x_{1}^{5}\end{array}\right),\left(\begin{array}{cccc}x_{3}^{3} & x_{7}^{3} & x_{3}^{4} & x_{7}^{4} \\ x_{7}^{10} & x_{1}^{8} & x_{7}^{9} & x_{1}^{7}\end{array}\right),\left(\begin{array}{cccc}x_{4}^{3} & x_{8}^{3} & x_{4}^{4} & x_{8}^{4} \\ x_{8}^{10} & x_{1}^{8} & x_{8}^{9} & x_{1}^{7}\end{array}\right),\left(\begin{array}{cccc}x_{3}^{5} & x_{5}^{5} & x_{3}^{6} & x_{5}^{6} \\ x_{5}^{9} & x_{1}^{10} & x_{5}^{10} & x_{1}^{9}\end{array}\right)$,

$\left(\begin{array}{cccc}x_{4}^{5} & x_{6}^{5} & x_{4}^{6} & x_{6}^{6} \\ x_{6}^{9} & x_{1}^{10} & x_{6}^{10} & x_{1}^{9}\end{array}\right),\left(\begin{array}{cccc}x_{3}^{7} & x_{7}^{7} & x_{3}^{8} & x_{7}^{8} \\ x_{7}^{2} & x_{3}^{3} & x_{7}^{1} & x_{3}^{4}\end{array}\right),\left(\begin{array}{cccc}x_{4}^{7} & x_{8}^{7} & x_{4}^{8} & x_{8}^{8} \\ x_{8}^{2} & x_{4}^{3} & x_{8}^{1} & x_{4}^{4}\end{array}\right),\left(\begin{array}{cccc}x_{1}^{9} & x_{5}^{9} & x_{1}^{10} & x_{5}^{10} \\ x_{7}^{4} & x_{1}^{2} & x_{7}^{3} & x_{1}^{1}\end{array}\right)$,

$\left(\begin{array}{cccc}x_{1}^{9} & x_{6}^{9} & x_{1}^{10} & x_{6}^{10} \\ x_{8}^{4} & x_{1}^{2} & x_{8}^{3} & x_{1}^{1}\end{array}\right),\left(\begin{array}{cccc}x_{3}^{5} & x_{7}^{7} & x_{3}^{6} & x_{7}^{8} \\ x_{7}^{3} & x_{3}^{10} & x_{7}^{4} & x_{3}^{9}\end{array}\right),\left(\begin{array}{cccc}x_{4}^{5} & x_{8}^{7} & x_{4}^{6} & x_{8}^{8} \\ x_{8}^{3} & x_{4}^{10} & x_{8}^{4} & x_{4}^{9}\end{array}\right),\left(\begin{array}{cccc}x_{1}^{7} & x_{9}^{9} & x_{1}^{8} & x_{9}^{10} \\ x_{6}^{3} & x_{1}^{4} & x_{5}^{3} & x_{1}^{3}\end{array}\right)$,

$\left(\begin{array}{cccc}x_{3}^{7} & x_{7}^{9} & x_{3}^{8} & x_{7}^{10} \\ x_{7}^{3} & x_{3}^{6} & x_{7}^{4} & x_{3}^{5}\end{array}\right),\left(\begin{array}{cccc}x_{4}^{7} & x_{8}^{9} & x_{4}^{8} & x_{8}^{10} \\ x_{8}^{3} & x_{4}^{6} & x_{8}^{4} & x_{4}^{5}\end{array}\right),\left(\begin{array}{cccc}x_{3}^{8} & x_{5}^{3} & x_{3}^{10} & x_{6}^{3} \\ x_{9}^{3} & x_{2}^{8} & x_{7}^{8} & x_{2}^{10}\end{array}\right),\left(\begin{array}{llll}x_{3}^{7} & x_{5}^{4} & x_{3}^{9} & x_{6}^{4} \\ x_{9}^{4} & x_{2}^{7} & x_{7}^{7} & x_{2}^{9}\end{array}\right)$,

$\left(\begin{array}{cccc}x_{3}^{8} & x_{7}^{3} & x_{3}^{10} & x_{8}^{3} \\ x_{5}^{6} & x_{2}^{8} & x_{9}^{3} & x_{2}^{10}\end{array}\right),\left(\begin{array}{cccc}x_{3}^{7} & x_{7}^{4} & x_{3}^{9} & x_{8}^{4} \\ x_{5}^{5} & x_{2}^{7} & x_{9}^{4} & x_{2}^{9}\end{array}\right),\left(\begin{array}{cccc}x_{4}^{8} & x_{5}^{3} & x_{4}^{10} & x_{6}^{3} \\ x_{9}^{3} & x_{2}^{10} & x_{8}^{8} & x_{2}^{8}\end{array}\right),\left(\begin{array}{cccc}x_{4}^{7} & x_{5}^{4} & x_{4}^{9} & x_{6}^{4} \\ x_{9}^{4} & x_{2}^{9} & x_{8}^{7} & x_{2}^{7}\end{array}\right)$,

$\left(\begin{array}{cccc}x_{4}^{8} & x_{7}^{3} & x_{4}^{10} & x_{8}^{3} \\ x_{6}^{6} & x_{2}^{10} & x_{9}^{3} & x_{2}^{8}\end{array}\right),\left(\begin{array}{cccc}x_{4}^{7} & x_{7}^{4} & x_{4}^{9} & x_{8}^{4} \\ x_{6}^{5} & x_{2}^{9} & x_{9}^{4} & x_{2}^{7}\end{array}\right),\left(\begin{array}{cccc}x_{1}^{8} & x_{9}^{3} & x_{1}^{10} & x_{9}^{4} \\ x_{5}^{3} & x_{2}^{8} & x_{6}^{3} & x_{2}^{7}\end{array}\right),\left(\begin{array}{cccc}x_{1}^{7} & x_{9}^{3} & x_{1}^{9} & x_{9}^{4} \\ x_{5}^{4} & x_{2}^{10} & x_{6}^{4} & x_{2}^{9}\end{array}\right)$.

Theorem 4.4. $K_{m} \otimes \overline{K_{n}}$ has an $L_{8}-$ decomposition if and only if $m n^{2}(m-1) \equiv 0(\bmod 16)$.

Proof. Necessity. Assume that $K_{m} \otimes \overline{K_{n}}$ admits an $L_{8^{-}}$decomposition. Then the number of edges in the graph $K_{m} \otimes \overline{K_{n}}$ is $\frac{m n^{2}(m-1)}{2}$ which should be divisible by 8 , the number of edges in $L_{8}$ i.e., $16 \mid m n^{2}(m-1)$ and hence $m n^{2}(\stackrel{2}{m}-1) \equiv 0(\bmod 16)$.

Sufficiency. We construct the required decomposition in five cases.

Case $(1) n \equiv 0(\bmod 4)$. 
Let $n=4 s$, for some $s>0$. Then we can write $K_{m} \otimes \overline{K_{n}}=\left(\frac{m(m-1)}{2}\right) K_{4 s, 4 s}$. Now, we get the desired decomposition by Theorem 2.7 .

Case $(2) m \equiv 0(\bmod 4), n \equiv 0(\bmod 2)$.

Subcase $(\boldsymbol{i}) m \equiv 0(\bmod 4), n \equiv 0(\bmod 4)$. Proof follows from Case $(1)$.

Subcase $($ ii $) m \equiv 0(\bmod 4), n \equiv 2(\bmod 4)$.

Let $m=4 s$ and $n=6,10$ for some $s>0$. Now we can write $K_{m} \otimes \overline{K_{n}}=s\left(K_{4} \otimes \overline{K_{n}}\right) \oplus \frac{s(s-1)}{2}\left(K_{4,4} \otimes \overline{K_{n}}\right)$ and the graphs $K_{4} \otimes \overline{K_{6}}, K_{4} \otimes \overline{K_{10}}$ can be viewed as $3 K_{6,12}, 3 K_{10,20}$, respectively. Therefore we get the desired decomposition, by Lemma 4.1 and the Theorem 2.7. Let $m=4 s$ and $n=4 t+2$ for some $s>$ $0, t>2$. Now we can write $K_{m} \otimes \overline{K_{n}}=\left(K_{4 s} \otimes \overline{K_{4(t-1)}}\right) \oplus\left(\frac{s(s-1)}{2}\right)\left(K_{4,4} \otimes \overline{K_{6}}\right) \oplus 4 s(4 s-1) K_{4(t-1), 6}$ $\oplus 3 s K_{6,12}$. We get the desired decomposition, by the Case (1), Lemma 4.1 and Theorem 2.7.

Case(3) $m \equiv 1(\bmod 4), n \equiv 0(\bmod 2)$.

Subcase $(i) m \equiv 1(\bmod 4), n \equiv 0(\bmod 4)$. Proof follows from Case $(1)$.

Subcase $($ ii $) m \equiv 1(\bmod 4), n \equiv 2(\bmod 4)$.

Let $m=4 s+1$ and $n=6,10$ for some $s>0$. Now we can write $K_{m} \otimes \overline{K_{n}}=\left(K_{4(s-1)} \otimes \overline{K_{n}}\right) \oplus K_{5} \otimes \overline{K_{n}}$ $\oplus(s-1)\left(K_{4,5} \otimes \overline{K_{n}}\right)$ and the graphs $K_{5} \otimes \overline{K_{6}}, K_{5} \otimes \overline{K_{10}}$ can be viewed as $5 K_{6,12}, 5 K_{10,20}$, respectively. Therefore we get the desired decomposition, by the Case(2), Lemmas 4.2, 4.3 and Theorem 2.7. Further, let $m=4 s+1$ and $n=4 t+2$ for some $s>0, t>2$. Now we can write $K_{m} \otimes \overline{K_{n}}=K_{4(s-1)} \otimes \overline{K_{4 t+2}}$ $\oplus K_{5} \otimes \overline{K_{4(t-1)}} \oplus(s-1)\left(K_{4,5} \otimes \overline{K_{4(t-1)}}\right) \oplus(s-1)\left(K_{4,5} \otimes \overline{K_{6}}\right) \oplus 20 s K_{4(t-1), 6} \oplus 5 K_{6,12}$. The $L_{8^{-}}$ decomposition of $1^{\text {st }}$ term of the above sum follows from Case (2), $2^{\text {nd }}$ and $3^{\text {rd }}$ term follows from Case (1) and the remaining terms of the above sum follows from the Lemma 4.2 and Theorem 2.7. Hence we get the desired decomposition.

Case(4) $m \equiv 0(\bmod 16)$.

Let $m=16 s$, for some $s>0$. We can write $K_{m} \otimes \overline{K_{n}}=s\left(K_{16} \otimes \overline{K_{n}}\right) \oplus\left(\frac{s(s-1)}{2}\right)\left(K_{16,16} \otimes \overline{K_{n}}\right)$. The desired decomposition follows from Lemmma 4.1 and Theorems 1.3, 2.7.

Case(5) $m \equiv 1(\bmod 16)$.

Desired decomposition follows from Theorem 1.3 and Lemma 4.1.

\section{Conclusion}

In this paper, we established necessary and sufficient conditions for the decomposition of tensor/wreath product of graphs into sunlet graphs of order 8 . Further, research on the existence of such decomposition of product graphs into sunlet graphs of higher order $r>8$ is under progress.

Acknowledgement: The first author thank the Department of Science and Technology, Government of India, New Delhi for its financial support through the Grant No.DST/INSPIRE Fellowship/2015/IF150211. The second author thank the University Grant Commission, Government of India, New Delhi for its support through the grant No.F.510/7/DRS-I/2016(SAP-I).

\section{References}

[1] A. D. Akwu, D. D. A. Ajayi, Decomposing certain equipartite graphs into Sunlet graphs of length 2p, AKCE Int. J. Graphs Combin. 13 (2016) 267-271.

[2] B. Alspach, The wonderful Walecki construction, Bull. Inst. Combin. Appl. 52 (2008) 7-20.

[3] B. Alspach, J. C. Bermond, D. Sotteau, Decomposition into cycles I: Hamilton decompositions, In: Cycles and rays (Montreal, PQ, 1987), Kluwer Academic Publishers, Dordrecht, (1990) 9-18.

[4] B. Alspach, H. Gavlas, Cycle decompositions of $K_{n}$ and $K_{n-1}$, J. Combin. Theory Ser. B 81(1) 
(2001) 77-99.

[5] R. Anitha, R. S. Lekshmi, N-sun decomposition of complete graphs and complete bipartite graphs, World Acad. Sci. Eng. Tech. 27 (2007) 262-266.

[6] R. Anitha, R.S. Lekshmi, N-sun decomposition of complete, complete bipartite and some Harary graphs, Int. J. Comput. Math. Sci. 2 (2008).

[7] J. A. Bondy, U. R. S. Murty, Graph theory with applications, The Macmillan Press Ltd, New York (1976).

[8] D. Bryant, Cycle decompositions of complete graphs, in Surveys in Combinatorics 2007, A. Hilton and J. Talbot (Editors), London Mathematical Society Lecture Note Series 346, Proceedings of the 21st British Combinatorial Conference, Cambridge University Press (2007) 67-97.

[9] D. Bryant, C. A. Rodger, Cycle decompositions, C.J. Colbourn, J.H. Dinitz (Eds.), The CRC Handbook of Combinatorial Designs (2nd edition), CRC Press, Boca Raton (2007) 373-382.

[10] D. Froncek, Decomposition of complete graphs into small graphs, Opuscula Math. 30(3) (2010) $277-280$.

[11] C. M. Fu, M. H. Huang, Y. L. Lin, On the existence of 5-sun systems, Discrete Math. 313(24) (2013) 2942-2950.

[12] C. M. Fu, N. H. Jhuang, Y. L. Lin, H. M. Sung, From steiner triple systems to 3-sun systems, Taiwanese J. Math. 16(2) (2012) 531-543.

[13] C. M. Fu, N. H. Jhuang, Y. L. Lin, H. M. Sung, On the existence of k-sun systems, Discrete Math. 312 (2012) 1931-1939.

[14] M. Gionfriddo, G. Lo Faro, S. Milici, A. Tripodi, On the existence of uniformly resolvable decompositions of $K$ into 1-factors and h-suns, Utilitas Mathematica 99 (2016) 331-339.

[15] A. J. W. Hilton, Hamiltonian decompositions of complete graphs, J. Combin.Theory B 36(2) (1984) $125-134$

[16] A. J. W. Hilton, C. A. Rodger, Hamiltonian decompositions of complete regular s-partite graphs, Discrete Math. 58 (1986) 63-78.

[17] Z. Liang, J. Guo, Decomposition of complete multigraphs into Crown graphs, J. Appl. Math. Comput. 32 (2010) 507-517.

[18] Z. Liang, J. Guo, J. Wang, On the Crown graph decompositions containing odd cycle, Int. J. Comb. Graph Theory Appl. 4 (2019) 1-23.

[19] C. Lin, T-W Shyu, A necessary and sufficient condition for the star decomposition of complete graphs, J. Graph Theory, 23 (1996) 361-364.

[20] R. Frucht, Graceful numbering of wheels and related graphs, Ann. New York Acad. Sci 319 (1979) 219-229.

[21] M. Sajna, Cycle decompositions III: Complete graphs and fixed length cycles, J. Combin. Des. 10 (2002) 27-78.

[22] D. Sotteau, Decomposition of $K_{m, n}\left(K_{m, n}^{*}\right)$ into cycles (circuits) of length $2 k$, J. Combin. Theory Ser. B 30(1) (1981) 75-81.

[23] M. Tarsi, Decomposition of complete multigraphs into stars, Discrete Mathematics 26(3) (1979) $273-278$.

[24] M. Tarsi, Decomposition of complete multigraph into simple paths: Nonbalanced Handcuffed designs, J. Combin. Theory Ser. A 34 (1983) 60-70.

[25] M. Truszczynski, Note on the decomposition of $\lambda K_{m, n}\left(\lambda K_{m, n}^{*}\right)$ into paths, Discrete Math. 55 (1985) 89-96.

[26] K. Ushio, S. Tazawa, S. Yamamoto, On claw-decomposition of complete multipartite graphs, Hiroshima Math. J. 8(1) (1978) 207-210.

[27] S. Yamamoto, H. Ikeda, S. Shige-Eda, K. Ushio, N. Hamada, On claw decomposition of complete graphs and complete bipartite graphs, Hiroshima Math. J. 5(1) (1975) 33-42. 